\title{
Upper bounds on the non-3-colourability threshold of random graphs
}

\author{
Nikolaos Fountoulakis ${ }^{1 *}$ and Colin McDiarmid ${ }^{2}$ \\ ${ }^{1}$ Mathematical Institute, University of Oxford, 24-29 St. Giles', Oxford OX1 3LB, UK. \\ 2 Department of Statistics, University of Oxford, 1 South Parks Road, Oxford OX1 3TG, UK.
}

received Oct 10, 2002, accepted Oct 28, 2002.

We present a full analysis of the expected number of 'rigid' 3-colourings of a sparse random graph. This shows that, if the average degree is at least 4.99, then as $n \rightarrow \infty$ the expected number of such colourings tends to 0 and so the probability that the graph is 3-colourable tends to 0 . (This result is tight, in that with average degree 4.989 the expected number tends to $\infty$.) This bound appears independently in Kaporis et al. [14]]. We then give a minor improvement, which shows in particular that the probability that the graph is 3-colourable tends to 0 if the average degree is at least 4.989 .

Keywords: sparse random graphs, 3-colourability, thresholds

\section{Introduction}

We are concerned with the 3-colourability of sparse random graphs. We consider the $\mathcal{G}_{n, m}$ model (also known as the uniform model), where $m=\lceil\theta n / 2\rceil$. This consists of all graphs with vertex set $V_{n}=$ $\{1,2, \ldots, n\}$ which have $m$ edges, where each of these graphs occurs with probability $\left(\begin{array}{c}n \\ 2 \\ m\end{array}\right){ }^{-1}$. Note that the expected degree of a vertex is $\theta+o(1)$ as $n \rightarrow \infty$. We say that a graph property holds asymptotically almost surely (aas) if the probability that the random graph of order $n$ has this property tends to 1 as $n \rightarrow \infty$.

The threshold for non-2-colourability (that is, for the existence of an odd circuit) is well understood, and is not sharp, see for example the recent survey of Molloy [118]. For $k>2$ our understanding of $k$-colourability is less complete. Let $\chi(G)$ denote the chromatic number of a graph $G$. Erdős in [9, 3] asked whether for each $k \geq 3$ there exists a constant $\theta_{k}$ such that for any $\varepsilon>0, \chi\left(\mathcal{G}_{n, m}\right) \leq k$ aas when $m \leq\left(\theta_{k} / 2-\varepsilon\right) n$, and $\chi\left(G_{n, m}\right)>k$ aas when $m \geq\left(\theta_{k} / 2+\varepsilon\right) n$. Note that $\theta_{k}$ would specify a critical average degree for the random graph. In the case of 3-colourability, the experiments in [13] suggest that $\theta_{3} \simeq 4.6$.

Recently Achlioptas and Friedgut [II] gave a partial answer to the above question. They showed that for each fixed $k \geq 3$, there exists a function $d_{k}(n)$ such that for any $\varepsilon>0, \chi\left(\mathcal{G}_{n, m}\right) \leq k$ aas when $m=m(n) \leq$ $\left(\frac{d_{k}(n)}{2}-\varepsilon\right) n$, and $\chi\left(\mathcal{G}_{n, m}\right)>k$ aas when $m \geq\left(\frac{d_{k}(n)}{2}+\varepsilon\right) n$. It is widely believed that $\lim _{n \rightarrow \infty} d_{k}(n)$ exists, but confirming this conjecture and determining the limit $\theta_{k}$ seems challenging. Let

$$
\theta_{k}^{-}=\liminf _{n \rightarrow \infty} d_{k}(n)=\sup \left\{\theta>0: \chi\left(\mathcal{G}_{n,[\theta n / 2\rceil}\right) \leq k \text { aas }\right\}
$$

and

$$
\theta_{k}^{+}=\limsup _{n \rightarrow \infty} d_{k}(n)=\inf \left\{\theta>0: \chi\left(\mathcal{G}_{n,\lceil\theta n / 2\rceil}\right)>k \text { aas }\right\} .
$$

\footnotetext{
${ }^{*}$ Research supported by EPSRC Studentship, Award No.: 00801424, and "Alexander S. Onassis" Public Benefit Foundation
} Cont. No.: W-112/2000. 
Then $\theta_{k}^{-} \leq \theta_{k}^{+}$and the two are equal if and only if the answer to the question of Erdós is positive.

Let us return to the case $k=3$. There are many results concerning lower bounds on $\theta_{3}^{-}$; in other words showing that for a certain value of $\theta$, we have $\chi\left(\mathcal{G}_{n, m}\right) \leq 3$ aas if $m=m(n) \leq \theta n / 2$, and so $\theta_{3}^{-} \geq \theta$ (see [18] for a survey on this topic).

There are fewer results concerning upper bounds on $\theta_{3}^{+}$, which is our interest here. These upper bounds are based on the following idea. For each graph $G$ on $V_{n}$ let $C(G)$ denote the set of all proper 3-colourings of $G$ using colours $1,2,3$; and let $C^{\prime}(G) \subseteq \mathcal{C}(G)$ satisfy $C^{\prime}(G) \neq \emptyset$ whenever $\mathcal{C}(G) \neq \emptyset$. We call such a family of colourings adequate. Let us denote $\left|C^{\prime}(G)\right|$ by $C^{\prime}(G)$ : we shall adopt such notation throughout the paper. By Markov's inequality,

$$
\mathbb{P}\left(\chi\left(\mathcal{G}_{n, m}\right) \leq 3\right) \leq \mathbb{E}\left[C^{\prime}\left(\mathcal{G}_{n, m}\right)\right]
$$

Of course equality holds here if $C^{\prime}(G) \leq 1$ always. The aim is to find a small adequate family which is simple enough to handle, and then to use (11) to yield an upper bound on $\theta_{3}^{+}$.

When we consider all possible 3-colourings, that is we take $C^{\prime}(G)=C(G)$ for each $G$, we find that $\mathbb{E}\left[C^{\prime}\left(G_{n, m}\right)\right] \rightarrow 0$ as $n \rightarrow \infty$ if $\theta=5.41$, and so $\theta_{3}^{+} \leq 5.41$. This basic first moment result was improved by Dunne and Zito in $[8]$ to $\theta_{3}^{+} \leq 5.2057$ by considering a certain adequate family; and then further improved by Achlioptas and Molloy [2] to $\theta_{3}^{+} \leq 5.044$ by considering a smaller adequate family, namely the 'rigid' 3 -colourings. The idea of considering such colourings came from the success of the method of 'locally maximum' satisfying truth assignments in [15] for investigating the unsatisfiability threshold for random 3-SAT problems, see the survey [18].

Definition 1 A proper 3-colouring with stable sets $S_{1}, S_{2}, S_{3}$ is called rigid if each vertex in $S_{2} \cup S_{3}$ is adjacent to some vertex in $S_{1}$, and each vertex in $S_{3}$ is adjacent to some vertex in $S_{2}$.

The above bound from [2] was obtained independently by the authors in [II]. [In much earlier work [17], Molloy and Reed took a different approach to improving the basic bound $\theta_{3}^{+} \leq 5.41$ : they showed that a random graph of average degree at least 5.142 is aas not 3-colourable by proving that the 3-core of such a random graph is aas not 3-colourable. We do not follow that approach here.] In recent work, Kaporis et al. [14] give a tighter estimate of the expected number of rigid 3-colourings, and obtain $\theta_{3}^{+} \leq 4.99$. We obtained this result independently and concurrently (see [11]), and present here a more complete analysis of the expected number of rigid 3-colourings, which shows in particular that the last bound cannot be improved, in the sense that with average degree 4.989 the expected number of rigid 3 -colourings tends to $\infty$.

Now, let us introduce our central theorem. Let $m=\lceil\theta n / 2\rceil$. For a graph $G$, let $\mathcal{R}(G)$ denote the set of rigid 3-colourings of $G$, and let $R(G)$ denote the cardinality of this set. For $\theta$ in an interval $\left[\theta_{l}, \theta_{u}\right]$, and for $\mathbf{x}$ in a domain $\mathcal{D}=\mathcal{D}(\theta) \subseteq[0,1]^{3}$, we shall introduce a function $h(\mathbf{x}, \theta)$ (defined in (18) below), and let $\mu(\theta)=\sup _{\mathbf{x} \in \mathcal{D}} h(\mathbf{x}, \theta)$.

Theorem 1.1 There exist positive real numbers $\theta_{0}<\theta_{1}$ such that:

1. The function $\mu(\theta)$ is continuous on $\left[\theta_{0}, \theta_{1}\right]$, and for every $\theta \in\left[\theta_{0}, \theta_{1}\right]$

$$
\mathbb{E}\left[R\left(\mathcal{G}_{n, m}\right)\right]=2^{\mu(\theta) n+O(\log n)},
$$

where $m=\lceil\theta n / 2\rceil$. Moreover, $\mu\left(\theta_{0}\right)>0$ and $\mu\left(\theta_{1}\right)<0$.

2. We have

$$
4.9893<\theta_{0}<\theta_{1}<4.9895 .
$$

In the above theorem as well as in what follows, the symbol log always refers to binary logarithm. In order to prove this result, one half of the battle is to show (2), and the other half is to show (3)). The main step in proving (3) is to show that for $\theta=4.9895$, we have $\mu(\theta)<0$. This involves considerable computation: we are more explicit about this side of matters than has been the custom in previous papers in this area. From the previous theorem and the Markov inequality (II) we obtain 
Corollary 1.2 Let $\theta=4.9895$ and $m=\lceil\theta n / 2\rceil$. Then there exists $\delta>0$ such that $\mathbb{P}\left[\chi\left(\mathcal{G}_{n, m}\right) \leq 3\right] \leq 2^{-\delta n}$, for $n$ sufficiently large.

Therefore, $\theta_{3}^{+} \leq 4.9895$. Working from the foundation provided by Theorem 1.1, we may try to improve the bound on $\theta_{3}^{+}$in different ways. One way involves an adequate subfamily of the rigid 3-colourings, the " $\Psi$-gadget-free" rigid 3-colourings - see the brief discussion in Section 7 . A second way involves considering the number of non-trivial tree components, and using the observation that any non-trivial tree has at least two rigid 3-colourings: this approach is described in Section 6 below, and yields the following theorem.

Theorem 1.3 Let $\theta=4.98887$ and $m=\lceil\theta n / 2\rceil$. Then there exists $\delta>0$ such that $\mathbb{P}\left[\chi\left(\mathcal{G}_{n, m}\right) \leq 3\right] \leq 2^{-\delta n}$, for $n$ sufficiently large.

Similar results can be deduced for the $\mathcal{G}_{n, p}$ model. In this model, again $V_{n}=\{1,2, \ldots, n\}$ is the set of vertices, but now each of the $\left(\begin{array}{l}n \\ 2\end{array}\right)$ possible edges appears independently with probability $p$. We find that if $\theta=4.98887$ and $p=\theta / n$, then there exists a $\delta>0$ such that $\mathbb{P}\left[\chi\left(\mathcal{G}_{n, p}\right) \leq 3\right] \leq 2^{-\delta n}$, for $n$ sufficiently large.

The main steps in the proof of Theorem 1.1 are as follows. We first give an exact formula for $\mathbb{E}\left[R\left(\mathcal{G}_{n, m}^{*}\right)\right]$, where $\mathcal{G}_{n, m}^{*}$ is a slight variation of the $\mathcal{G}_{n, m}$ model; see Lemma 2.1 below. We show that we can discard the 'tails' of the sum that appears there, and give good approximations to the remaining 'central' terms. These terms involve probabilities $p(k, \alpha, n, \theta)$, which are investigated in Section 3. The probabilities can be written as a sum, where the summands involve binomial coefficients and certain 'balls-and-bins' probabilities. Again we show that we can discard the tails in the sums, and give good approximations to the remaining central terms, now involving Stirling numbers of the second kind. We use known asymptotic expressions for these Stirling numbers, thus expressing the summands as terms like $2^{h(\mathbf{x}, \theta) n}$. This yields an approximation for $\mathbb{E}\left[R\left(\mathcal{G}_{n, m}^{*}\right)\right]$ as $2^{\mu(\theta) n+O(\log n)}$ (see (22) below) and we then obtain (2). This part of the proof is completed in Section 4 .

The remaining work to prove Theorem $[1.1$ is largely numerical, and is described in Section 5 . The main task is to show that for $\theta=4.9895$, we have $\mu(\theta)<0$. We show that, for this specific $\theta, h(\mathbf{x}, \theta)$ is concave over its domain $\mathcal{D}$. We find numerically a first approximation for a point which gives the maximum value of $h$ inside this area. We define a very fine grid in a box around this point, and find a grid point $\hat{\mathbf{x}}$ where the maximum value of $h$ on the grid is attained. Then, we determine an upper bound for $h$ on the surface of the box (by computing values of $h$ and its partial derivatives on a fine grid and using concavity). We find that this bound is less than the value of $h$ at $\hat{\mathbf{x}}$, and so we deduce that the box contains the maximum of $h$ over $\mathcal{D}$. Further computations handle the region inside the box.

After completing the proof of Theorem 1.1 as described above, in Section 6 we prove Theorem 1.3, and then we make some brief concluding remarks in Section 7 . Some details from earlier proofs are given in the Appendices.

\section{Starting the proofs}

For the sake of simplicity, we carry out the probability calculations in the $\mathcal{G}_{n, m}^{*}$ model. In this model, we form the random graph by choosing at random $m$ times, each time independently, uniformly and with replacement, an edge out of the $\left(\begin{array}{l}n \\ 2\end{array}\right)$ possible 2-subsets of $V_{n}=\{1, \ldots, n\}$. We ignore any repetitions of an edge, so the random graph may have less than $m$ edges. Our results transfer easily to the $\mathcal{G}_{n, m}$ model - see Lemma 4.3 below. Every probability, unless otherwise stated, is meant to be taken over the $\mathcal{G}_{n, m}^{*}$ model.

Let

$$
\mathrm{D}=\{(k, \alpha): 0 \leq k \leq 1,0 \leq \alpha \leq k\},
$$

and for each positive integer $n$ let

$$
\mathrm{D}^{(n)}=\mathrm{D} \cap \frac{1}{n} \mathbb{Z}^{2}
$$

For each $(k, \alpha) \in \mathrm{D}$ we define $\phi(k, \alpha)=k(1-\alpha)-(k-\alpha)^{2}$. 
Let $\mathcal{C}(n)$ be the set of all 3-colourings of $V_{n}$, i.e. the set of all possible mappings from $V_{n}$ into $\{1,2,3\}$, or equivalently the set of all partitions of $V_{n}$ into three sets $S_{1}, S_{2}$ and $S_{3}$ (some of them possibly empty). Also, for each positive integer $n$ and each $(k, \alpha) \in \mathrm{D}^{(n)}$, let $\mathcal{C}(k, \alpha, n)$ denote the set of all partitions of $V_{n}$ into three sets $S_{1}, S_{2}, S_{3}$, where $\left|S_{1}\right|=\alpha n$ and $\left|S_{1}\right|+\left|S_{2}\right|=k n$. Let

$$
p(k, \alpha, n, m)=\mathbb{P}[S \text { is rigid } \mid S \text { is proper }],
$$

where $S \in \mathcal{C}(k, \alpha, n)$. Note that the above quantity depends only on the sizes of the independent sets induced by $S$. Recall that $R(G)$ denotes the number of rigid 3-colourings of $G$.

Lemma 2.1 For all positive integers $n$ and $m$ with $0<m \leq\left(\begin{array}{l}n \\ 2\end{array}\right)$,

$$
\mathbb{E}\left[R\left(\mathcal{G}_{n, m}^{*}\right)\right]=\left(1-\frac{1}{n}\right)^{-m} \sum_{(k, \alpha) \in \mathbb{D}^{(n)}}\left(\begin{array}{c}
n \\
k n
\end{array}\right)\left(\begin{array}{c}
k n \\
\alpha n
\end{array}\right)(2 \phi(k, \alpha))^{m} p(k, \alpha, n, m) .
$$

Proof. By the linearity of the expected value, we have

$$
\begin{aligned}
\mathbb{E}\left[R\left(G_{n, m}^{*}\right)\right] & =\sum_{S \in \mathcal{C}(n)} \mathbb{P}[S \text { is rigid }] \\
& =\sum_{S \in \mathcal{C}(n)} \mathbb{P}[S \text { is rigid } \mid S \text { is proper }] \cdot \mathbb{P}[S \text { is proper }] .
\end{aligned}
$$

Let us take a fixed colouring $S$ with stable sets $S_{1}, S_{2}, S_{3}$, where $s_{1}=\left|S_{1}\right|=\alpha n, s_{2}=\left|S_{2}\right|=\beta n$ and $s_{3}=\left|S_{3}\right|=\gamma n$, and where $k=\alpha+\beta$. Set

$$
e(S)=s_{1} s_{2}+s_{1} s_{3}+s_{2} s_{3} .
$$

Hence,

$$
\frac{e(S)}{n^{2}}=(\alpha \beta+\beta \gamma+\gamma \alpha)=\phi(k, \alpha)
$$

Thus,

$$
\mathbb{P}[S \text { is proper }]=\left(\frac{e(S)}{\left(\begin{array}{l}
n \\
2
\end{array}\right)}\right)^{m}=(2 \phi(k, \alpha))^{m}\left(1-\frac{1}{n}\right)^{-m} .
$$

Now notice that the family $\mathcal{C}(k, \alpha, n)$ consists of exactly $\left(\begin{array}{c}n \\ k n\end{array}\right) \cdot\left(\begin{array}{c}k n \\ \alpha n\end{array}\right)$ colourings. So, rephrasing the sum in (5) in terms of $k$ and $\alpha$ we obtain the following:

$$
\begin{aligned}
\mathbb{E}\left[R\left(\mathcal{G}_{n, m}^{*}\right)\right] & =\sum_{S \in \mathcal{C}(n)} \mathbb{P}[S \text { is rigid }] \\
& =\left(1-\frac{1}{n}\right)^{-m} \sum_{(k, \alpha) \in \mathrm{D}^{(n)}}\left(\begin{array}{c}
n \\
k n
\end{array}\right)\left(\begin{array}{c}
k n \\
\alpha n
\end{array}\right)(2 \phi(k, \alpha))^{m} p(k, \alpha, n, m) .
\end{aligned}
$$

The above lemma is our starting point. We next check that in Lemma 2.1 we may ignore the extreme values of $\alpha$ and $k$. We will split the sum there into two pieces. Let

$$
\mathrm{D}_{1}=\{(k, \alpha): \alpha \geq 0.2, k \leq 0.8, k-\alpha \geq 0.2\}
$$

(which corresponds to $\alpha, \beta, \gamma \geq 0.2$ ); and let $\mathrm{D}_{1}^{(n)}=\mathrm{D}_{1} \cap \frac{1}{n} \mathbb{Z}^{2}$. Moreover, doing some elementary calculations, we obtain the following: 
Lemma 2.2 The function $\phi(k, \alpha)$ is continuous and concave on $\mathrm{D}$, for each $(k, \alpha) \in \mathrm{D}$ we have $0 \leq$ $\phi(k, \alpha) \leq 1 / 3$, and

$$
\begin{aligned}
& \sup _{(k, \alpha) \in \mathrm{D} \backslash \mathrm{D}_{1}} \phi(k, \alpha)=0.32, \\
& \min _{(k, \alpha) \in \mathrm{D}_{1}} \phi(k, \alpha)=0.28 .
\end{aligned}
$$

Let

$$
\mathcal{S}_{1}=\left(1-\frac{1}{n}\right)^{-m} \sum_{(k, \alpha) \in \mathrm{D}_{1}^{(n)}}\left(\begin{array}{c}
n \\
k n
\end{array}\right)\left(\begin{array}{c}
k n \\
\alpha n
\end{array}\right)(2 \phi(k, \alpha))^{m} p(k, \alpha, n, m),
$$

and let

$$
\mathcal{S}_{2}=\left(1-\frac{1}{n}\right)^{-m} \sum_{(k, \alpha) \in \mathrm{D}^{(n)} \backslash \mathrm{D}_{1}^{(n)}}\left(\begin{array}{c}
n \\
k n
\end{array}\right)\left(\begin{array}{c}
k n \\
\alpha n
\end{array}\right)(2 \phi(k, \alpha))^{m} p(k, \alpha, n, m),
$$

so that by Lemma 2.1

$$
\mathbb{E}\left[R\left(\mathcal{G}_{n, m}^{*}\right)\right]=\mathcal{S}_{1}+\mathcal{S}_{2} .
$$

We will see that the second 'error' term here is negligible for the relevant values of $m$; and then we may focus on the first term.

Lemma 2.3 Let $4.98 \leq a \leq b$. Then there exist $\delta>0$ and $n_{0} \in \mathbb{N}$ such that for $\theta \in[a, b], n \geq n_{0}$ and $m=\lceil\theta n / 2\rceil$ we have:

$$
\mathcal{S}_{2} \leq 2^{-\delta n}
$$

Proof. By Lemma 2.2, we have

$$
\sigma=\sup _{(k, \alpha) \in \mathrm{D} \backslash \mathrm{D}_{1}} \phi(k, \alpha)=0.32
$$

Thus, we have

$$
\begin{aligned}
\mathcal{S}_{2} & \leq \sum_{(k, \alpha) \in \mathrm{D}^{(n)} \backslash \mathrm{D}_{1}^{(n)}}\left(\begin{array}{c}
n \\
k n
\end{array}\right)\left(\begin{array}{c}
k n \\
\alpha n
\end{array}\right)\left(\frac{n}{n-1} 2 \phi(k, \alpha)\right)^{m} \\
& \leq 3^{n}\left(\frac{n}{n-1} 2 \sigma\right)^{\frac{\theta n}{2}} \\
& =\left(\frac{n}{n-1}\right)^{\theta n / 2}\left(3(2 \sigma)^{\frac{\theta}{2}}\right)^{n}=O\left(\left(3(2 \sigma)^{\theta / 2}\right)^{n}\right) .
\end{aligned}
$$

But $3(0.64)^{\frac{4.98}{2}}<1$, and the lemma follows.

The following standard lemma on approximating binomial coefficients may be proved using Stirling's formula:

Lemma 2.4 Let $0<\delta<1 / 2$. Then uniformly over $\delta n \leq r \leq(1-\delta) n$ we have

$$
\left(\begin{array}{l}
n \\
r
\end{array}\right)=\Theta\left(n^{-1 / 2}\right)\left(\frac{n}{r}\right)^{r}\left(\frac{n}{n-r}\right)^{n-r}=\Theta\left(n^{-1 / 2}\right) 2^{H(r / n)},
$$

where $H(x)=-x \log x-(1-x) \log (1-x)$ for $0 \leq x \leq 1$ (when $x=0$ or $x=1$, then $H(x)=0$ ) is the entropy function.

For $m=\lceil\theta n / 2\rceil$, we set

$$
p(k, \alpha, n, \theta)=p(k, \alpha, n, m)
$$

(the use of the same letter $p$ should not cause confusion). Directly from the definition (ㄱ) and the last lemma, we have: 
Lemma 2.5 Uniformly over $\theta$ in any interval $[a, b]$ with $a>0$,

$$
\mathcal{S}_{1}=\Theta\left(n^{-1}\right) \sum_{(k, \alpha) \in D_{1}^{(n)}}\left(2^{H(k)+k H(\alpha / k)}(2 \phi(\alpha, k))^{\theta / 2}\right)^{n} p(k, \alpha, n, \theta) .
$$

In the next section we consider the term $p(k, \alpha, n, \theta)$.

\section{Calculations for $p(k, \alpha, n, \theta)$}

In this section, we derive an asymptotic formula for $p(k, \alpha, n, \theta)$, which was defined in (9) to be equal to $\mathbb{P}[S$ is rigid $\mid S$ is proper $]$, where $S \in \mathcal{C}(k, \alpha, n), m=\lceil\theta n / 2\rceil$ and we are working in the $\mathcal{G}_{n, m}^{*}$ model. See Lemma B.2 below.

For positive integers $t \geq r$, we let $p(t, r)$ denote the probability that, when we throw $t$ balls uniformly at random into $r$ bins, each bin ends up non-empty. Consider $(k, \alpha) \in \mathrm{D}^{(n)}$. Let $L^{(n)}=L^{(n)}(k, \alpha, \theta)=$ $\left\{\lambda: \frac{n(1-\alpha)}{m} \leq \lambda \leq 1-\frac{n(1-k)}{m}\right\} \cap\left(\frac{1}{m} \mathbb{Z}\right)$. Then

$$
p(k, \alpha, n, \theta)=\sum_{\lambda \in L^{(n)}} b(\lambda m ; m, p) p(\lambda m, n(1-\alpha)) p((1-\lambda) m, n(1-k)),
$$

where

$$
p=1-((1-k)(k-\alpha)) / \phi(k, \alpha)
$$

and $b(\lambda m ; m, p)$ is the probability that a random variable distributed according to the binomial distribution $\operatorname{Bi}(m, p)$ is equal to $\lambda m$. To see this, observe first that, conditioning on $S$ being a proper 3-colouring, the random variable that determines the number of edges between $S_{1}$ and $S_{2} \cup S_{3}$ is binomially distributed, namely it is $\operatorname{Bi}(m, p)$, where $p$ is defined in (I1). Once we have specified the number of edges between $S_{1}$ and $S_{2} \cup S_{3}$ (and, therefore, the number of edges between $S_{2}$ and $S_{3}$ as well), the probability that $S$ is rigid is exactly the probability that each vertex in $S_{2} \cup S_{3}$ is adjacent to some vertex in $S_{1}$ and each vertex in $S_{3}$ is adjacent to some vertex in $S_{2}$. Note that for each edge, say, between $S_{1}$ and $S_{2} \cup S_{3}$, each vertex in $S_{2} \cup S_{3}$ has the same probability to be the endvertex of it. The same holds for the edges between $S_{2}$ and $S_{3}$. Thus, we can think of this as a random throwing of balls into bins; each ball corresponding to an edge and each bin corresponding to a vertex. Note that we have two independent such random experiments. This observation yields (10).

\subsection{Discarding the tails}

We next check that we may discard the extreme values of $\lambda$ in (10). This is a technical exercise for which we need one preliminary lemma.

Lemma 3.1 For positive integers $t>r$

$$
\frac{t}{2(t-r)} p(t-1, r) \leq p(t, r) \leq \frac{t}{t-r} p(t-1, r)
$$

Proof. Let $W(t, r)$ be the set of all arrangements of $t$ balls into $r$ bins leaving no empty bins and let $w(t, r)$ be its cardinality. What we want to prove will follow from the following inequality:

$$
\frac{r t}{2(t-r)} w(t-1, r) \leq w(t, r) \leq \frac{r t}{t-r} w(t-1, r) .
$$

To prove (12), consider ordered pairs of balls and bins, i.e. if $T$ is the set of balls and $R$ the set of bins, take the Cartesian product of them $P=T \times R$. Each such pair $(b, B)$, where $b \in T$ and $B \in R$, corresponds to the fact that the ball $b$ is in bin $B$. For each such pair arrange the remaining $t-1$ balls into the $r$ bins leaving no empty bins. Thus, we form the set $\mathcal{W}=\{(p, w): p \in P, w \in W(t-1, r)\}$. Note that we have 
a surjective mapping from the set $\mathcal{W}$ onto the set $W(t, r)$. Clearly, $\mathcal{W}$ is of cardinality $r t w(t-1, r)$. The mapping induces a natural partition on this set and each of the parts, which is the set of pairs that are mapped to a specific arrangement of $t$ balls into $r$ bins without leaving any empty bins, is of cardinality equal to the number of balls which are not the only ball in their bin, which is at least $t-r$ and at most $2(t-r)$. Thus, (12) has been established. Therefore,

$$
\frac{t}{2(t-r)} \frac{w(t-1, r)}{r^{t-1}} \leq \frac{w(t, r)}{r^{t}} \leq \frac{t}{t-r} \frac{w(t-1, r)}{r^{t-1}},
$$

and the lemma follows.

For $(k, \alpha) \in \mathrm{D}_{1}$, let

$$
L_{1}=L_{1}(k, \alpha, \theta)=\left\{\lambda: \frac{2(1-\alpha)}{\theta}(1+0.24) \leq \lambda \leq 1-\frac{2(1-k)}{\theta}(1+0.065)\right\},
$$

and let

$$
L_{1}^{(n)}=L_{1}^{(n)}(k, \alpha, \theta)=L_{1} \cap\left(\frac{1}{m} \mathbb{Z}\right) .
$$

(The extra terms 0.24 and 0.065 here are to exclude extreme values which, as we shall see shortly, are negligible, but which would cause awkwardness later.)

It is convenient to restrict $\theta$ to a range $\left[\theta_{l}, \theta_{u}\right]$, as we need to obtain approximations uniformly over $\theta$. We let

$$
\theta_{l}=4.98 \quad \theta_{u}=4.99
$$

Lemma 3.2 Uniformly over $\theta \in\left[\theta_{l}, \theta_{u}\right]$ and $(k, \alpha) \in \mathrm{D}_{1}^{(n)}$,

$$
p(k, \alpha, n, \theta)=\Theta(1) \sum_{\lambda \in L_{1}^{(n)}} b(\lambda m ; m, p) p(\lambda m, n(1-\alpha)) p((1-\lambda) m, n(1-k)) .
$$

\section{(Recall that $p$ is defined in (11).)}

Proof. Within the proof, let $f(\lambda)$ be the general term in the sum in equation (10). We will compare the term $f(\lambda)$, for some $\lambda$ which will be specified later, with the adjacent term $f(\lambda-1 / m)$. Note that $f(\lambda)=b(\lambda m ; m, p) p(t, r) p\left(m-t, r^{\prime}\right)$, where $r=n(1-\alpha)$ and $r^{\prime}=n(1-k)$, and $t=\lambda m$.

We consider the "lower" tail first. Assume that $t=n(1-\alpha)+\lfloor\eta n(1-\alpha)\rfloor$, for some $\eta>0$. By Lemma B.] we have

$$
p(t-1, r) p\left(m-t+1, r^{\prime}\right) \leq 2\left(\frac{t-r}{t}\right)\left(\frac{m-t+1}{m-t+1-r^{\prime}}\right) p(t, r) p\left(m-t, r^{\prime}\right) .
$$

Also

$$
b(t-1 ; m, p)=\frac{\lambda m}{m(1-\lambda)+1} \frac{1-p}{p} b(t ; m, p) .
$$

Thus, we obtain

$$
f\left(\lambda-\frac{1}{m}\right) \leq 2\left(\frac{t-r}{t}\right)\left(\frac{m-t+1}{m-t+1-r^{\prime}}\right) \frac{\lambda m}{m(1-\lambda)+1} \frac{1-p}{p} f(\lambda) .
$$

Using the fact that $(k, \alpha) \in \mathrm{D}_{1}$, straightforward verification shows that for $\eta=0.25$ and for $n$ sufficiently large the factor on the right hand side is less than $1 / 2$ (in fact it is less than 0.45 ), for any $\theta \in\left[\theta_{l}, \theta_{u}\right]$. (This 
is the case because the factor is increasing with respect to $\eta$; see [10] for the details.) Therefore, the sum of the terms for $t$ from $n(1-\alpha)$ up to $n(1-\alpha)+\lfloor 0.25(1-\alpha) n\rfloor-1$ can be bounded as follows:

$$
\sum_{t=n(1-\alpha)}^{n(1-\alpha)+\lfloor 0.25(1-\alpha) n\rfloor-1} f\left(\frac{t}{m}\right) \leq f\left(\frac{n(1-\alpha)+\lfloor 0.25(1-\alpha) n\rfloor}{m}\right),
$$

by the geometric sum formula.

Following the same treatment, we can bound the other tail of the sum. Here, assume that $m-t=$ $n(1-k)+\lfloor\eta(1-k) n\rfloor$. Thus, $t=m-n(1-k)-\lfloor\eta(1-k) n\rfloor$. From Lemma 3.1 and (15), we obtain

$$
f(\lambda) \leq \frac{t}{t-r} \frac{2\left(m-t+1-r^{\prime}\right)}{m-t+1} \frac{(1-\lambda) m+1}{\lambda m} \frac{p}{1-p} f\left(\lambda-\frac{1}{m}\right) .
$$

Using the fact that $(k, \alpha) \in \mathrm{D}_{1}$, one can see that for $\eta=0.07$ and for $n$ sufficiently large the factor on the right hand side is less than $1 / 2$ (in fact it is less than 0.49 ), for any $\theta \in\left[\theta_{l}, \theta_{u}\right]$. As in the previous case, this expression is increasing with respect to $\eta$. Therefore, the sum of the terms for $t$ from $m-n(1-k)-$ $\lfloor 0.07(1-k) n\rfloor+1$ up to to $m-n(1-k)$ can be bounded as follows:

$$
\sum_{t=m-n(1-k)-\lfloor 0.07(1-k) n\rfloor+1}^{m-n(1-k)} f\left(\frac{t}{m}\right) \leq f\left(\frac{m-n(1-k)-\lfloor 0.07(1-k) n\rfloor}{m}\right),
$$

by the geometric sum formula.

Now, the lemma follows from the above observations along with the fact that each term is non-negative, which means that removing a few terms from the sum gives a lower bound on it.

\subsection{Introducing Stirling numbers of the second kind}

For positive integers $t \geq r$ the Stirling number of the second kind $S(t, r)$ is defined to be $1 / r$ ! times the number of surjective functions from a set of cardinality $t$ to a set of cardinality $r$. Thus

$$
p(t, r)=\frac{r ! S(t, r)}{r^{t}} .
$$

Hence, we may rewrite Lemma 3.2 as follows:

Lemma 3.3 Uniformly over $\theta \in\left[\theta_{l}, \theta_{u}\right]$ and $(k, \alpha) \in \mathrm{D}_{1}^{(n)}$, we have:

$$
\begin{aligned}
& p(k, \alpha, n, \theta)= \\
&= \Theta(1) \sum_{\lambda \in L_{1}^{(n)}}\left(\begin{array}{c}
\lceil\theta n / 2\rceil \\
\lambda\lceil\theta n / 2\rceil
\end{array}\right)\left(\frac{(1-k)(k-\alpha)}{\phi(k, \alpha)}\right)^{\left\lceil\frac{\theta n}{2}\right\rceil(1-\lambda)}\left(1-\frac{(1-k)(k-\alpha)}{\phi(k, \alpha)}\right)^{\lambda\left\lceil\frac{\theta n}{2}\right\rceil} \times \\
& \frac{(n(1-\alpha)) ! S(\lambda\lceil\theta n / 2\rceil, n(1-\alpha))}{(n(1-\alpha))^{\lambda\left\lceil\frac{\theta n}{2}\right\rceil}} \frac{(n(1-k)) ! S((1-\lambda)\lceil\theta n / 2\rceil, n(1-k))}{(n(1-k))^{\left\lceil\frac{\theta n}{2}\right\rceil(1-\lambda)}} .
\end{aligned}
$$

\subsection{Asymptotics for Stirling numbers of the second kind}

An essential part of our probability calculations involves asymptotic expressions for the Stirling numbers of the second kind. We need some preliminary definitions and results, see for example [20].

For $0<u \leq 1$ let

$$
E_{u}(x)=1-e^{-x}-u x
$$

For $0<u<1$ let $x_{0}(u)$ be the unique positive root of $E_{u}(x)=0$. (See Figure 11.) Note that $E_{1}(x)$ has the unique root $x=0$ : we let $x_{0}(1)=0$. Then $x_{0}(u)$ is a continuous function on $(0,1]$, and

$$
x_{0}(u)+1-1 / u>0
$$




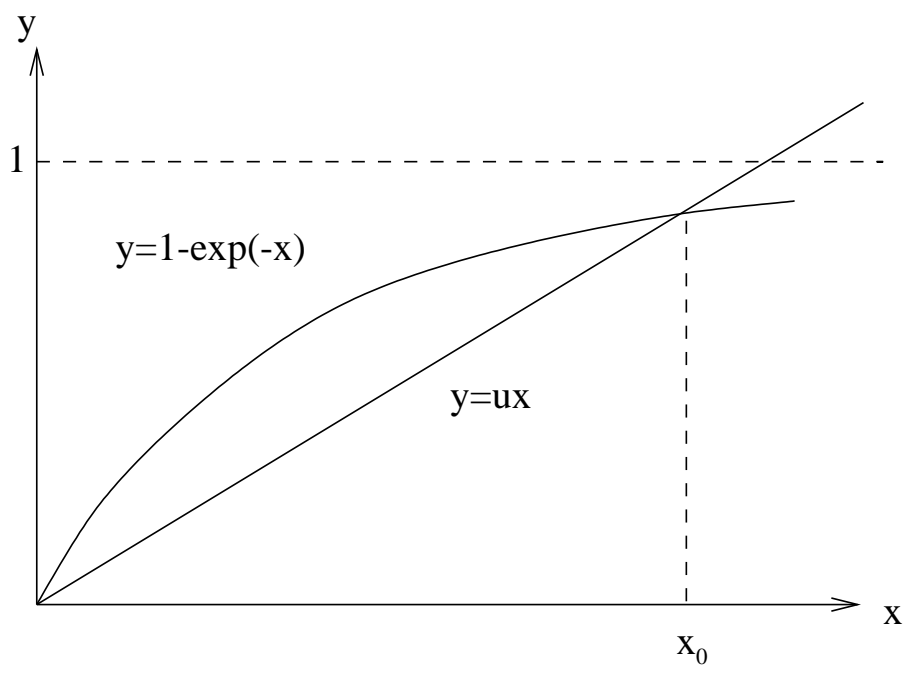

Fig. 1: The function $x_{0}(u)$

for each $0<u<1$.

For $0<u<1$ let

$$
f(u)=\left(\frac{1-u}{x_{0}(u)+1-1 / u}\right)^{1 / 2},
$$

and let $f(1)=0$. Then $f$ is a continuous function on $(0,1]$, and $0 \leq f(u) \leq 1$ (see [7]). In Appendix $\mathrm{A}$, we give a positive lower bound on $f(u)$.

It is shown in [20] that, for positive integers $t>r$,

$$
S(t, r)=\left(e^{x_{0}}-1\right)^{r}\left(x_{0}(u)\right)^{-t} e^{-(t-r)}(t-r)^{(t-r)}\left(\begin{array}{l}
t \\
r
\end{array}\right) f(u)(1+\varepsilon(t, r)),
$$

where $u=r / t$ and $\max _{0<r<t}|\varepsilon(t, r)| \rightarrow 0$ as $t \rightarrow \infty$.

\subsection{The estimate}

Recall that $\mathrm{D}_{1}$ and $L_{1}$ are defined in (6) and (13) above. Let

$$
\mathcal{D}=\mathcal{D}(\theta)=\left\{(k, \alpha, \lambda):(k, \alpha) \in \mathrm{D}_{1}, \lambda \in L_{1}(k, \alpha, \theta)\right\},
$$

and let

$$
\mathcal{D}^{(n)}=\mathcal{D}^{(n)}(\theta)=\mathcal{D} \cap\left(\left(\frac{1}{n} \mathbb{Z}^{2}\right) \times\left(\frac{1}{m} \mathbb{Z}\right)\right)=\left\{(k, \alpha, \lambda):(k, \alpha) \in \mathrm{D}_{1}^{(n)}, \lambda \in L_{1}^{(n)}(k, \alpha, \theta)\right\} .
$$

For $\theta \in\left[\theta_{l}, \theta_{u}\right]$ and $(k, \alpha, \lambda) \in \mathcal{D}$, let

$$
\begin{aligned}
P(k, \alpha, \lambda, \theta)= & (1-k)^{-\frac{\theta}{2}} 2^{\frac{\theta}{2} H(\lambda)}\left(\frac{1-k}{1-\alpha}\right)^{\frac{\lambda \theta}{2}}\left(e^{x_{1}}-1\right)^{(1-\alpha)}\left(e^{x_{2}}-1\right)^{(1-k)}\left(x_{1}\right)^{-\frac{\lambda \theta}{2}}\left(x_{2}\right)^{-\frac{\theta}{2}(1-\lambda)} \times \\
& e^{-\frac{\theta}{2}}\left(\frac{\lambda \theta}{2}\right)^{\frac{\theta \lambda}{2}}\left(\frac{\theta(1-\lambda)}{2}\right)^{\frac{\theta(1-\lambda)}{2}}\left(\frac{(1-k)(k-\alpha)}{\phi(k, \alpha)}\right)^{\frac{\theta}{2}(1-\lambda)}\left(1-\frac{(1-k)(k-\alpha)}{\phi(k, \alpha)}\right)^{\frac{\lambda \theta}{2}},
\end{aligned}
$$

where $x_{1}=x_{0}\left(\frac{2(1-\alpha)}{\theta \lambda}\right)$ and $x_{2}=x_{0}\left(\frac{2(1-k)}{\theta(1-\lambda)}\right)$. We shall prove the following lemma: 
Lemma 3.4 Uniformly over $\theta \in\left[\theta_{l}, \theta_{u}\right]$ and $(k, \alpha) \in \mathrm{D}_{1}$, we have:

$$
p(k, \alpha, n, \theta)=\Theta\left(n^{-1 / 2}\right) \sum_{\lambda \in L_{1}^{(n)}}\{P(k, \alpha, \lambda, \theta)\}^{n} .
$$

Proof. In what follows, the "error" term $\lceil\theta n / 2\rceil-\theta n / 2$ yields a $\Theta(1)$ factor for each term of the sum in Lemma 3.3, since $(k, \alpha) \in \mathrm{D}_{1}^{(n)}$. We use Lemma 2.4 to give asymptotic expressions for the binomial coefficients. Note that since $(k, \alpha) \in \mathrm{D}_{1}$ the assumptions of Lemma 2.4 are satisfied. This is also true for the coefficient that involves $\theta$, since $\theta$ is assumed to be in a closed and bounded interval not containing 0 . Thus, using (16) and Stirling's approximation for the factorials, Lemma 3.3 implies the following:

$$
p(k, \alpha, n, \theta)=\Theta\left(n^{-1 / 2}\right) \sum_{\lambda \in L_{1}^{(n)}}\left\{P^{\prime}(k, \alpha, \lambda, \theta)\right\}^{n},
$$

where, for $\theta \in\left[\theta_{l}, \theta_{u}\right]$ and $(k, \alpha, \lambda) \in \mathcal{D}$,

$$
\begin{aligned}
& P^{\prime}(k, \alpha, \lambda, \theta)= \\
&=\frac{n^{(2-\alpha-k)}(1-\alpha)^{(1-\alpha)}(1-k)^{(1-k)} e^{-(2-\alpha-k)}}{n^{\frac{\theta}{2}}(1-k)^{\frac{\theta}{2}}} 2^{\frac{\theta}{2} H(\lambda)}\left(\frac{1-k}{1-\alpha}\right)^{\frac{\lambda \theta}{2}} \times \\
& \quad n^{\left(\frac{\theta \lambda}{2}+\alpha-1\right)} t(1-\alpha, \lambda \theta / 2) n^{\left(\frac{\theta}{2}-\frac{\theta \lambda}{2}-1+k\right)} t(1-k,(1-\lambda) \theta / 2) \times \\
& \quad f\left(\frac{2(1-\alpha)}{\theta \lambda}\right) f\left(\frac{2(1-k)}{\theta(1-\lambda)}\right)(1-p)^{\frac{\theta}{2}(1-\lambda)} p^{\frac{\lambda \theta}{2}} \\
&= \frac{(1-\alpha)^{(1-\alpha)}(1-k)^{(1-k)} e^{-(2-\alpha-k)}}{(1-k)^{\frac{\theta}{2}}} 2^{\frac{\theta}{2} H(\lambda)}\left(\frac{1-k}{1-\alpha}\right)^{\frac{\lambda \theta}{2}} f\left(\frac{2(1-\alpha)}{\theta \lambda}\right) f\left(\frac{2(1-k)}{\theta(1-\lambda)}\right) \times \\
& t(1-\alpha, \lambda \theta / 2) t(1-k,(1-\lambda) \theta / 2)(1-p)^{\frac{\theta}{2}(1-\lambda)} p^{\frac{\lambda \theta}{2}},
\end{aligned}
$$

where

$$
t(x, y)=\left(e^{x_{0}}-1\right)^{y}\left(x_{0}\right)^{-x} e^{-(x-y)}(x-y)^{(x-y)}\left(\frac{x}{y}\right)^{y}\left(\frac{x}{x-y}\right)^{(x-y)},
$$

where $x_{0}=x_{0}(x / y)$ and where $p$ is defined in (11). Doing some calculations, we obtain:

$$
\begin{aligned}
P^{\prime}(k, \alpha, \lambda, \theta)= & \\
= & (1-k)^{-\frac{\theta}{2}} 2^{\frac{\theta}{2} H(\lambda)}\left(\frac{1-k}{1-\alpha}\right)^{\frac{\lambda \theta}{2}}\left(e^{x_{1}}-1\right)^{(1-\alpha)}\left(e^{x_{2}}-1\right)^{(1-k)}\left(x_{1}\right)^{-\frac{\lambda \theta}{2}}\left(x_{2}\right)^{-\frac{\theta}{2}(1-\lambda)} e^{-\frac{\theta}{2}} \times \\
& f\left(\frac{2(1-\alpha)}{\theta \lambda}\right) f\left(\frac{2(1-k)}{\theta(1-\lambda)}\right)\left(\frac{\lambda \theta}{2}\right)^{\frac{\theta \lambda}{2}}\left(\frac{\theta(1-\lambda)}{2}\right)^{\frac{\theta(1-\lambda)}{2}}(1-p)^{\frac{\theta}{2}(1-\lambda)} p^{\frac{\lambda \theta}{2}} \\
= & P(k, \alpha, \lambda, \theta) f\left(\frac{2(1-\alpha)}{\theta \lambda}\right) f\left(\frac{2(1-k)}{\theta(1-\lambda)}\right),
\end{aligned}
$$

where $x_{1}=x_{0}\left(\frac{2(1-\alpha)}{\theta \lambda}\right)$ and $x_{2}=x_{0}\left(\frac{2(1-k)}{\theta(1-\lambda)}\right)$. For the elementary but tedious calculations see Appendix B.

Also, note that by (27) in Appendix A, the monotonicity of the lower bound for $f(u)$, where $u=\frac{2(1-\alpha)}{\theta \lambda}$ or $u=\frac{2(1-k)}{\theta(1-\lambda)}$, and the fact that $(k, \alpha) \in \mathrm{D}_{1}$, it follows that in both cases the function $f$ is at least

$$
\sqrt{\frac{1-\frac{2}{5 \theta}}{x_{0}\left(\frac{2}{5 \theta}\right)}}
$$


and so the two factors including $f$ in the expression above yield a $\Theta(1)$ term. This concludes the proof of the lemma.

\section{Proof of Theorem 1.1 and Corollary 1.2}

Recall that $\theta_{l}, \theta_{u}$ were introduced in (14) and $\mathcal{D}$ was defined in (17). For $\theta \in\left[\theta_{l}, \theta_{u}\right]$ and $(k, \alpha, \lambda) \in \mathcal{D}$, let

$$
\begin{aligned}
h(k, \alpha, \lambda, \theta)= & H(k)+k H\left(\frac{\alpha}{k}\right)+\frac{\theta}{2}-\frac{\theta}{2} \log (e)+\frac{\theta}{2} \log \left(\frac{\theta}{2}\right) \\
& +\frac{\theta}{2}(-\lambda \log \lambda-(1-\lambda) \log (1-\lambda)+(1-\lambda) \log (1-k) \\
& +(1-\lambda) \log (k-\alpha)+\lambda \log \alpha+\lambda \log (1-\alpha)) \\
& +(1-k) \log \left(e^{x_{2}}-1\right)-\frac{\theta}{2}(1-\lambda) \log \left(x_{2}\right)-\frac{\theta(1-\lambda)}{2} \log (1-k)+\frac{\theta(1-\lambda)}{2} \log (1-\lambda) \\
& +(1-\alpha) \log \left(e^{x_{1}}-1\right)-\frac{\lambda \theta}{2} \log \left(x_{1}\right)-\frac{\lambda \theta}{2} \log (1-\alpha)+\frac{\lambda \theta}{2} \log \lambda,
\end{aligned}
$$

where $x_{1}=x_{0}\left(\frac{2(1-\alpha)}{\theta \lambda}\right)$ and $x_{2}=x_{0}\left(\frac{2(1-k)}{\theta(1-\lambda)}\right)$. (Recall that the function $x_{0}(u)$ was defined at the start of Subsection 3.3 ) We will prove the following:

Lemma 4.1 Uniformly over $\theta \in\left[\theta_{l}, \theta_{u}\right]$,

$$
\mathcal{S}_{1}=\Theta\left(n^{-3 / 2}\right) \sum_{(k, \alpha, \lambda) \in \mathcal{D}^{(n)}} 2^{h(k, \alpha, \lambda, \theta) n} .
$$

Proof. Lemmas 2.5 and 3.4 imply that uniformly over $\theta \in\left[\theta_{l}, \theta_{u}\right]$ :

$$
\begin{aligned}
\mathcal{S}_{1} & =\Theta\left(n^{-3 / 2}\right) \sum_{(k, \alpha, \lambda) \in \mathcal{D}^{(n)}}\left(2^{H(k)} 2^{k H\left(\frac{\alpha}{k}\right)}(2 \phi(k, \alpha))^{\frac{\theta}{2}} P(k, \alpha, \lambda, \theta)\right)^{n} \\
& =\Theta\left(n^{-3 / 2}\right) \sum_{(k, \alpha, \lambda) \in \mathcal{D}^{(n)}}\left(2^{h(k, \alpha, \lambda, \theta)}\right)^{n},
\end{aligned}
$$

since for $(k, \alpha, \lambda) \in \mathcal{D}$,

$$
\begin{aligned}
2^{H(k)} & 2^{k H\left(\frac{\alpha}{k}\right)}(2 \phi(k, \alpha))^{\frac{\theta}{2}} P(k, \alpha, \lambda, \theta)= \\
= & 2^{H(k)} 2^{k H\left(\frac{\alpha}{k}\right)}(2 \phi(k, \alpha))^{\frac{\theta}{2}} 2^{\frac{\theta}{2} H(\lambda)}(1-k)^{-\frac{\theta}{2}}\left(\frac{1-k}{1-\alpha}\right)^{\frac{\lambda \theta}{2}}\left(e^{x_{1}}-1\right)^{(1-\alpha)}\left(e^{x_{2}}-1\right)^{(1-k)} \times \\
& \left(x_{1}\right)^{-\frac{\lambda \theta}{2}}\left(x_{2}\right)^{-\frac{\theta}{2}(1-\lambda)} e^{-\frac{\theta}{2}}\left(\frac{\lambda \theta}{2}\right)^{\frac{\theta \lambda}{2}}\left(\frac{\theta(1-\lambda)}{2}\right)^{\frac{\theta(1-\lambda)}{2}}(1-p)^{\frac{\theta}{2}(1-\lambda)} p^{\frac{\lambda \theta}{2}} \\
= & 2^{H(k)} 2^{k H\left(\frac{\alpha}{k}\right)} 2^{\frac{\theta}{2}} 2^{\frac{\theta}{2} H(\lambda)}((1-k)(k-\alpha))^{\frac{\theta}{2}(1-\lambda)}(\alpha(1-\alpha))^{\frac{\lambda \theta}{2}}(1-k)^{-\frac{\theta(1-\lambda)}{2}}(1-\alpha)^{-\frac{\lambda \theta}{2}} \times \\
& \left(e^{x_{1}}-1\right)^{(1-\alpha)}\left(e^{x_{2}}-1\right)^{(1-k)}\left(x_{1}\right)^{-\frac{\lambda \theta}{2}}\left(x_{2}\right)^{-\frac{\theta}{2}(1-\lambda)} e^{-\frac{\theta}{2}}\left(\frac{\lambda \theta}{2}\right)^{\frac{\theta \lambda}{2}}\left(\frac{\theta(1-\lambda)}{2}\right)^{\frac{\theta(1-\lambda)}{2}} \\
= & 2^{h(k, \alpha, \lambda, \theta)},
\end{aligned}
$$

where $p$ is defined in (III). Hence, the lemma has been established.

By the last lemma,

$$
\mathcal{S}_{1}=c(n, \theta)\left(\max _{(k, \alpha, \lambda) \in \mathcal{D}^{(n)}}\left\{2^{h(k, \alpha, \lambda, \theta)}\right\}\right)^{n}=c(n, \theta) 2^{\mu(\theta, n) n},
$$


where $c(n, \theta)=\Omega\left(n^{-3 / 2}\right), c(n, \theta)=O\left(n^{3 / 2}\right)$ and

$$
\mu(\theta, n)=\max _{(k, \alpha, \lambda) \in \mathcal{D}^{(n)}}\{h(k, \alpha, \lambda, \theta)\} .
$$

Let $\mathcal{D}^{(\infty)}=\liminf \mathcal{D}^{(n)}$ and note that it is the set of rationals contained in $\mathcal{D}$ and, therefore, it is dense inside it. Then, we have

$$
\lim _{n \rightarrow \infty} \mu(\theta, n)=\mu(\theta)=\sup _{(k, \alpha, \lambda) \in \mathcal{D}^{(\infty)}} h(k, \alpha, \lambda, \theta)=\max _{(k, \alpha, \lambda) \in \mathcal{D}} h(k, \alpha, \lambda, \theta)
$$

since for each fixed $\theta$ the function $h(k, \alpha, \lambda, \theta)$ is continuous on $\mathcal{D}$, which is a compact subset of $\mathbb{R}^{3}$. In fact, we can say a little more than this. We know that $h(k, \alpha, \lambda, \theta)$ attains its maximum at an internal point of $\mathcal{D}$, say $\mathbf{x}^{*}$. Note that this is a stationary point and one can also see that $h$ is differentiable on $\mathcal{D}$ and its derivatives are continuous. The latter implies that for any $\varepsilon>0$ there exists an open ball $U$ containing $\mathbf{x}^{*}$ where $\|\nabla h\|<\varepsilon$. For $n$ sufficiently large, there is a point $\mathbf{x}_{n} \in \mathcal{D}^{(n)} \cap U$ with

$$
\left\|\mathbf{x}_{n}-\mathbf{x}^{*}\right\|^{2} \leq\left(2\left(\frac{1}{2 n}\right)^{2}+\left(\frac{1}{m}\right)^{2}\right) \leq\left(\frac{1}{2}+\frac{4}{\theta_{l}^{2}}\right) n^{-2},
$$

and then

$$
\mu(\theta, n) \geq \mu(\theta)-\varepsilon\left(\frac{1}{2}+\frac{4}{\theta_{l}^{2}}\right)^{1 / 2} n^{-1},
$$

by the Mean Value Theorem. Hence,

$$
\sup _{\theta \in\left[\theta_{l}, \theta_{u}\right]} n(\mu(\theta)-\mu(\theta, n))=o(1) .
$$

This fact along with (19) imply the following:

Lemma 4.2 Uniformly over $\theta \in\left[\theta_{l}, \theta_{u}\right]$,

$$
S_{1}=2^{\mu(\theta) n+O(\log n)} .
$$

Since $h(k, \alpha, \lambda, \theta)$ is continuous on its domain $\mathcal{D}$, and $h$ as a function of $\theta$ is also continuous, the function $\mu(\theta)$ is continuous as well. As we shall see later, for $\theta_{0}=4.9893$, we have

$$
\mu\left(\theta_{0}\right)>0 .
$$

The numerical investigation in the next section shows that

$$
\mu\left(\theta_{2}\right)<0,
$$

for $\theta_{2}=4.9895$. By Lemma 2.3 , there exists $\tilde{\delta}>0$ such that $\mathcal{S}_{2}=O\left(2^{-\tilde{\delta} n}\right)$, uniformly over $\theta \in\left[\theta_{l}, \theta_{u}\right]$. We set $\delta^{\prime}=\min \left\{\tilde{\delta},-\mu\left(\theta_{2}\right)\right\}$. Let

$$
\theta_{1}=\inf \left\{\theta \geq \theta_{0}: \mu(\theta) \leq-\delta^{\prime} / 2\right\} .
$$

Note that (20) and (21) imply that $\theta_{0}<\theta_{1}<\theta_{2}$. Thus, we have $\mu\left(\theta_{1}\right)=-\delta^{\prime} / 2$ and $\mu(\theta) \geq-\delta^{\prime} / 2$ for each $\theta \in\left[\theta_{0}, \theta_{1}\right]$. Therefore, Lemma 4.2 implies that uniformly over $\theta \in\left[\theta_{0}, \theta_{1}\right]$, we have

$$
\mathbb{E}\left[R\left(\mathcal{G}_{n, m}^{*}\right)\right]=2^{\mu(\theta) n+O(\log n)}+\mathcal{S}_{2}=2^{\mu(\theta) n+O(\log n)}+O\left(2^{-\delta^{\prime} n}\right)=2^{\mu(\theta) n+O(\log n)},
$$

that is

$$
\mathbb{E}\left[R\left(\mathcal{G}_{n, m}^{*}\right)\right]=2^{\mu(\theta) n+O(\log n)} .
$$

Now, to establish Theorem [1.1, i.e. to show that this result in fact is also true in the $\mathcal{G}_{n, m}$ model, we have to do a little more work. We prove the following: 
Lemma 4.3 Let $\theta_{l} \leq b<\infty$. Uniformly over $\theta \in\left[\theta_{l}, b\right]$ we have

$$
\mathbb{E}\left[R\left(\mathcal{G}_{n, m}\right)\right]=\Theta(1) \mathbb{E}\left[R\left(\mathcal{G}_{n, m}^{*}\right)\right],
$$

where $m=\lceil\theta n / 2\rceil$.

Proof. To see the one direction note that

$$
\mathbb{E}\left[R\left(\mathcal{G}_{n, m}^{*}\right)\right] \geq \mathbb{E}\left[R\left(\mathcal{G}_{n, m}^{*}\right)|| E\left(\mathcal{G}_{n, m}^{*}\right) \mid=m\right] \mathbb{P}\left[\left|E\left(\mathcal{G}_{n, m}^{*}\right)\right|=m\right]=\mathbb{E}\left[R\left(\mathcal{G}_{n, m}\right)\right] \mathbb{P}\left[\left|E\left(\mathcal{G}_{n, m}^{*}\right)\right|=m\right] .
$$

Here, $E(G)$ denotes the set of edges of a graph $G$. Recall that $\ln (1-x) \geq-\frac{x}{1-x} \geq-2 x$, for $0 \leq x \leq 1 / 2$. Hence, if $2 m \leq\left(\begin{array}{l}n \\ 2\end{array}\right)$,

$$
\mathbb{P}\left[\left|E\left(\mathcal{G}_{n, m}^{*}\right)\right|=m\right]=\prod_{i=0}^{m-1}\left(1-\frac{i}{\left(\begin{array}{c}
n \\
2
\end{array}\right)}\right) \geq \exp \left(-2 \frac{\left(\begin{array}{c}
m \\
2
\end{array}\right)}{\left(\begin{array}{l}
n \\
2
\end{array}\right)}\right) .
$$

This expression is bounded away from 0 uniformly for $\theta$ in the closed interval $\left[\theta_{l}, b\right]$.

The other direction is a little more tricky. To see this note as before that

$$
\mathbb{P}\left[S \in \mathcal{R}\left(\mathcal{G}_{n, m}\right)\right]=\mathbb{P}\left[S \text { is proper for } \mathcal{G}_{n, m}\right] \mathbb{P}\left[S \in \mathcal{R}\left(\mathcal{G}_{n, m}\right) \mid S \text { is proper for } \mathcal{G}_{n, m}\right] .
$$

By Lemma 2.2, we have $e(S)=n^{2} \phi(k, \alpha) \geq 0.28 n^{2}$, for a colouring $S \in \mathcal{C}(k, \alpha, n)$, where $(k, \alpha) \in \mathrm{D}_{1}$, whence we obtain $e(S) / m^{2} \geq \eta>0$, for some $\eta$ (depending only on $b$ ). Thus (once $2 m \leq e(S)$ ),

$$
\left(1-\frac{m}{e(S)}\right)^{m}=\exp \left(m \ln \left(1-\frac{m}{e(S)}\right)\right) \geq \exp \left(-2 m^{2} / e(S)\right) \geq e^{-2 / \eta} \text {. }
$$

Hence, for such an $S$, we have

$$
\begin{aligned}
\mathbb{P}\left[S \text { is proper for } \mathcal{G}_{n, m}\right] & =\frac{e(S)}{\left(\begin{array}{l}
n \\
2
\end{array}\right)} \frac{e(S)-1}{\left(\begin{array}{l}
n \\
2
\end{array}\right)-1} \cdots \frac{e(S)-m+1}{\left(\begin{array}{l}
n \\
2
\end{array}\right)-m+1} \\
& \geq\left(\left(\frac{e(S)}{\left(\begin{array}{l}
n \\
2
\end{array}\right)}\right)\left(1-\frac{m}{e(S)}\right)\right)^{m} \\
& \geq e^{-2 / \eta} \mathbb{P}\left[S \text { is proper for } \mathcal{G}_{n, m}^{*}\right] .
\end{aligned}
$$

On the other hand,

$$
\mathbb{P}\left[S \in \mathcal{R}\left(\mathcal{G}_{n, m}\right) \mid S \text { is proper for } \mathcal{G}_{n, m}\right] \geq \mathbb{P}\left[S \in \mathcal{R}\left(\mathcal{G}_{n, m}^{*}\right) \mid S \text { is proper for } \mathcal{G}_{n, m}^{*}\right],
$$

since adding edges to a proper 3-colouring increases the probability that this is rigid. Therefore,

$$
\mathbb{P}\left[S \in \mathcal{R}\left(\mathcal{G}_{n, m}^{*}\right)\right] \leq e^{2 / \eta} \mathbb{P}\left[S \in \mathcal{R}\left(\mathcal{G}_{n, m}\right)\right] .
$$

Finally, by Lemma 2.3 , since $\theta \geq 4.98$,

$$
\begin{aligned}
\mathbb{E}\left[R\left(\mathcal{G}_{n, m}^{*}\right)\right] & =(1+o(1)) \sum_{(k, \alpha) \in \mathrm{D}_{1}^{(n)}} \sum_{S \in \mathcal{C}(k, \alpha, n)} \mathbb{P}\left[S \in \mathcal{R}\left(\mathcal{G}_{n, m}^{*}\right)\right] \\
& \leq(1+o(1)) e^{2 / \eta} \sum_{(k, \alpha) \in \mathrm{D}_{1}^{(n)}} \sum_{S \in \mathcal{C}(k, \alpha, n)} \mathbb{P}\left[S \in \mathcal{R}\left(\mathcal{G}_{n, m}\right)\right] \\
& \leq(1+o(1)) e^{2 / \eta} \mathbb{E}\left[R\left(\mathcal{G}_{n, m}\right)\right] .
\end{aligned}
$$

Thus, Lemma 4.3 along with (22) and inequalities (20) and (21) conclude the proof of Theorem [1.1. Corollary 1.2 follows immediately. 


\section{Numerical Computations}

Let us quickly dispose of the easy result (20). As above, let $\theta_{0}=4.9893$. Also, we set $k=0.698, \alpha=0.362$ and $\lambda=0.691$. We find using Maple with 10 digits precision that $h\left(k, \alpha, \lambda, \theta_{0}\right)>0$. In fact, to one digit it equals $5 \times 10^{-6}$. Moreover, for the same values of $k, \alpha$ and $\lambda$ and for $\theta=4.989, h(k, \alpha, \lambda, \theta)$ equals $7 \times 10^{-5}$, to one digit, and so $\mu(4.989)>0$ (yielding the result stated in brackets in the abstract). These values for $k, \alpha, \lambda$ were found using the Complex method [6]: for details of this and other computational matters see [10].

To complete the proof of Theorem [1.1, and thus of Corollary [1.2, it remains to establish (21). Let $h=h(k, \alpha, \lambda)=h\left(k, \alpha, \lambda, \theta_{2}\right)$, where as above $\theta_{2}=4.9895$. Then $h$ is continuous over $\mathcal{D}$, and we shall see in Appendix $\mathbb{Q}$ that it is strictly concave over the interior of $\mathcal{D}$. Thus, if $\mathcal{C} \subseteq \mathcal{D}$ and $\mathbf{y} \in C^{o}$ (the interior of $\mathcal{C}$ ) are such that $h(\mathbf{y})>h(\mathbf{x})$ for every $\mathbf{x}$ in the boundary of $\mathcal{C}$, then $h$ has a unique maximum point $\mathbf{x}^{*}$ over $\mathcal{D}$ and $\mathbf{x}^{*} \in \mathcal{C}^{o}$. Using concavity, we can estimate numerically where the maximum of $h$ is located, and give an upper bound on $h$ over this domain, as follows.

From an initial approximation to the maximum in $\mathcal{D}$, using the Complex method [5] (see also [6]), our attention is directed to the cube $\mathcal{C} \subseteq \mathcal{D}$, where $0.6980 \leq k \leq 0.6981,0.3622 \leq \alpha \leq 0.3623$ and $0.6910 \leq \lambda \leq 0.6911$. Divide the surface of the cube $\mathcal{C}$ into squares of side $s=5 \times 10^{-6}$. For a square centred at a, by the concavity of $h$ we obtain

$$
h(\mathbf{b}) \leq h(\mathbf{a})+(s / \sqrt{2})\|\nabla h(\mathbf{a})\|
$$

for each point $\mathbf{b}$ in the square. By checking each square, we find that $h(\mathbf{x}) \leq-3.937721 \times 10^{-5}$ for each point $\mathbf{x}$ on the surface of the cube $\mathcal{C}$. But there is a point $\mathbf{y}$ inside $\mathcal{C}$ with $h(\mathbf{y})$ strictly greater than this bound. More specifically, we may define a cubic grid inside $C$ each cube having side equal to $5 \times 10^{-6}$. The maximum value we find by searching this grid is equal to $-3.937414 \times 10^{-5}$ and it is strictly greater than the upper bound on $h$ on the surface of $\mathcal{C}$. Since $h$ is concave on $\mathcal{D}$, it follows as noted above that $h$ attains its maximum over $\mathcal{D}$ inside $\mathcal{C}$.

Now we obtain an upper bound on $h$ inside $\mathcal{C}$, using the aforementioned grid. By concavity, for each point $\mathbf{b}$ in the sub-cube with its centre located at $\mathbf{a}$ and edge of length equal to $s$, we have

$$
h(\mathbf{b}) \leq h(\mathbf{a})+\frac{\sqrt{3} s}{2}\|\nabla h(\mathbf{a})\| .
$$

By checking through each sub-cube, we find that $h(\mathbf{x})$ is less than $-3.9 \times 10^{-5}$, for each $\mathbf{x} \in \mathcal{C}$, and thus for each $\mathbf{x} \in \mathcal{D}$.

\section{Proof of Theorem 1.3}

Let $t(G)$ denote the number of components of the graph $G$ that are non-trivial trees (that is, trees with at least one edge).

Lemma 6.1 For any $t \geq 0$ and any positive integers $n$ and $m$ with $0 \leq m \leq\left(\begin{array}{l}n \\ 2\end{array}\right)$,

$$
\mathbb{P}\left[\chi\left(\mathcal{G}_{n, m}\right) \leq 3\right] \leq 2^{-t} \mathbb{E}\left[R\left(\mathcal{G}_{n, m}\right)\right]+\mathbb{P}\left[t\left(\mathcal{G}_{n, m}\right)<t\right] .
$$

Proof. Any non-trivial tree has at least 2 rigid 3-colourings. Thus, for a graph $G$, if $\chi(G) \leq 3$ and $t(G) \geq t$, then $R(G) \geq 2^{t}$. Hence,

$$
\mathbf{1}_{\{\chi(G) \leq 3\} \cap\{t(G) \geq t\}} \leq 2^{-t} R(G) .
$$

Now, we apply this result to $\mathcal{G}_{n, m}$ and take expectations. We obtain:

$$
\mathbb{P}\left[\left(\chi\left(\mathcal{G}_{n, m}\right) \leq 3\right) \wedge\left(t\left(\mathcal{G}_{n, m}\right) \geq t\right)\right] \leq 2^{-t} \mathbb{E}\left[R\left(\mathcal{G}_{n, m}\right)\right],
$$

and the lemma follows. 
For $\theta>0$ let

$$
\tau(\theta)=\sum_{t=2}^{\infty} \frac{\theta^{t-1} e^{-t \theta} t^{t-2}}{t !}
$$

and $m=\lceil\theta n / 2\rceil$. We shall use standard methods to prove:

Lemma 6.2 Let $\theta>0$. For any $\varepsilon>0$ there exists $\delta_{1}>0$ such that

$$
\mathbb{P}\left[t\left(\mathcal{G}_{n, m}\right) \leq \tau(\theta) n-\varepsilon n\right]=O\left(e^{-\delta_{1} n}\right) .
$$

Proof. Let $T$ be a tree on vertices $1, \ldots, t$, where $t$ is constant. Then

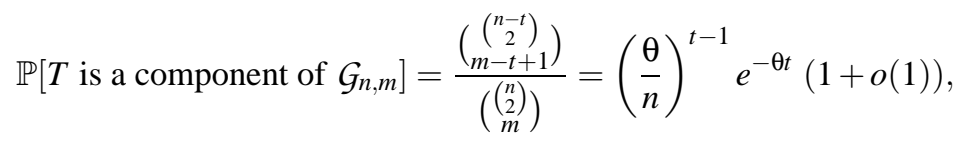

by standard approximations. Now we may multiply by $\left(\begin{array}{l}n \\ t\end{array}\right) t^{t-2}$ to see that the expected number of tree components with $t$ vertices in $\mathcal{G}_{n, m}$ is $(1+o(1)) n \theta^{t-1} e^{-\theta t} t^{t-2} / t$ !. Since the number of tree components of $\mathcal{G}_{n, m}$ with at least $t$ vertices is at most $n / t$, it follows that (see also [4] p.96):

$$
\mathbb{E}\left[t\left(\mathcal{G}_{n, m}\right)\right]=n\left(\sum_{t=2}^{\infty} \theta^{t-1} e^{-\theta t} t^{t-2} / t !\right)(1+o(1))=n \cdot \tau(\theta)(1+o(1)) .
$$

The following lemma will complete the proof, since if $G^{\prime}$ is obtained from $G$ by adding an edge and deleting an edge then $\left|t(G)-t\left(G^{\prime}\right)\right| \leq 2$.

The following lemma is a special case of Theorem 7.4 of [16] .

Lemma 6.3 Let $f$ be a function on graphs such that, if $G^{\prime}$ is obtained from $G$ by adding an edge and deleting an edge, then $\left|f(G)-f\left(G^{\prime}\right)\right| \leq c$. Let $\mu=\mathbb{E}\left[f\left(\mathcal{G}_{n, m}\right)\right]$. Then for any $x \geq 0$

$$
\mathbb{P}\left[f\left(\mathcal{G}_{n, m}\right)-\mu \geq x\right] \leq \exp \left(-2 x^{2} / m c^{2}\right)
$$

and

$$
\mathbb{P}\left[f\left(\mathcal{G}_{n, m}\right)-\mu \leq-x\right] \leq \exp \left(-2 x^{2} / m c^{2}\right) .
$$

Proof. Given an $m$-tuple $x$ of distinct edges of $K_{n}$, let $G(x)$ be the graph on $\{1, \ldots, n\}$ with edges those mentioned in $x$ (ignoring the order), and let $\tilde{f}(x)=f(G(x)) / c$. Then $|\tilde{f}(x)-\tilde{f}(y)| \leq 1$ if $x$ and $y$ differ in exactly one co-ordinate, or if they differ in exactly two co-ordinates and the values there are swapped. Thus we may apply Theorem 7.4 of [16], see also Example 7.3 there.

Therefore, setting $x=\tau(\theta) n-\varepsilon n$, for some $\varepsilon>0$ which will be specified later, in Lemma 6.1, and using also Lemmas 6.2, 4.2 and 4.3 along with equation (8) we obtain the following:

$$
\begin{aligned}
\mathbb{P}\left[\chi\left(\mathcal{G}_{n, m}\right) \leq 3\right] & \leq 2^{-n \tau(\theta)+\varepsilon n} \mathbb{E}\left[R\left(\mathcal{G}_{n, m}\right)\right]+O\left(e^{-\delta_{1} n}\right) \\
& =2^{\varepsilon n} 2^{-n \tau(\theta)+\Theta(1)}\left(2^{n \mu(\theta)+O(\log n)}+\mathcal{S}_{2}\right)+O\left(e^{-\delta_{1} n}\right) .
\end{aligned}
$$

We now fix $\theta=4.98887$.

From the proof of Lemma 2.3 we may see that $\mathcal{S}_{2} \leq 1$, for $n$ sufficiently large. We keep the definition of the region $\mathcal{D}$ unchanged. Inside $\mathcal{D}$, we may perform numerical investigations similar to those in the case of the rigid 3-colourings. We consider the same sub-cube $C$ as before, and find again that $h(k, \alpha, \lambda, \theta)$ attains its maximum inside $\mathcal{C}$. We can check through the same family of sub-cubes and see that the maximum value $\mu(\theta)$ of $h(k, \alpha, \lambda, \theta)$ over $\mathcal{D}$ satisfies $\mu(\theta)>0$, but $\tau(\theta)-\mu(\theta) \geq 10^{-5}$. Then by (23) with $\varepsilon=\frac{\tau(\theta)-\mu(\theta)}{2}$, we obtain:

$$
\mathbb{P}\left[\chi\left(\mathcal{G}_{n, m}\right) \leq 3\right] \leq 2^{-(\varepsilon+o(1)) n}+O\left(e^{-\delta_{1} n}\right) .
$$

Choosing $\delta<\min \left\{\varepsilon, \delta_{1} \log e\right\}$, we conclude the proof of Theorem 1.3 . 


\section{Concluding remarks}

We considered the adequate family consisting of the rigid 3-colourings of a graph, and investigated carefully the expected number of such colourings in the random graph $\mathcal{G}_{n, m}$. We thus obtained an upper bound on the non-3-colourability threshold $\theta_{3}^{+}$, which appears independently in [114]. We then improved this upper bound slightly, by taking into account the number of non-trivial tree components.

Let us sketch now some related ideas that also will improve the upper bound slightly. When we considered the number of non-trivial tree components in Section 6, in the proof of Lemma 6.1] we used the fact that $R(T) \geq 2$ for any non-trivial tree $T$. We can be more precise; for example $R(T) \geq 3$ unless $T$ is a star. By computing the value of $R(T)$ for each 'small' non-trivial tree (say those having at most 5 vertices), and then following the general approach in Section 6 , it is possible to obtain a slight improvement on Theorem [1.3] (see [10] for further details).

We may also consider an adequate subfamily of the rigid 3-colourings of a graph $G$, namely the leftmost 3 -colourings. These are the proper 3-colourings $S_{1}, S_{2}, S_{3}$ where $\left|S_{3}\right|$ is minimal and, subject to this, $\left|S_{2}\right|$ is minimal. Note that any such 3-colouring must be rigid and, further, this family is adequate. Unfortunately it seems to be hard to study leftmost 3-colourings, but we can work with related families such as those defined in terms of " $\Psi$-gadgets".

Given a 3-colouring $S_{1}, S_{2}, S_{3}$ of a graph $G$, a $\Psi_{12}$-gadget is a component of the subgraph induced on $S_{1} \cup S_{2}$, which is a star with centre in $S_{1}$ and at least 2 leaves (which must belong to $S_{2}$ ). We may define $\Psi_{13}-$ and $\Psi_{23}$-gadgets similarly. Call a rigid 3-colouring $\Psi$-gadget free if there are no $\Psi_{12}$ or $\Psi_{13}$ or $\Psi_{23}$ gadgets. Note that these 3-colourings form an adequate family, since each leftmost 3-colouring is $\Psi$-gadget free. By analysing such families of 3-colourings we may reduce the upper bound on $\theta_{3}^{+}$slightly - see [II]] and [110]. 


\section{References}

[1] D. Achlioptas and E. Friedgut. A sharp threshold for $k$-colourability. Random Structures \& Algorithms, 14(1):63-70, 1999.

[2] D. Achlioptas and M. Molloy. Almost all graphs with 2.522n edges are not 3-colorable. Electronic Journal of Combinatorics, 6 (1), 1999.

[3] N. Alon, J.H. Spencer, and P. Erdös. The Probabilistic Method. J. Wiley \& Sons, New York, 1992.

[4] B. Bollobás. Random Graphs. Academic Press, London, 1985.

[5] M.J. Box. A new method of constrained optimisation and a comparison with other methods. The Comp. Journal, 8:42-52, 1965.

[6] B. D. Bunday. Basic Optimisation Methods. Edward Arnold, London, 1984.

[7] O. Dubois and Y. Boufkhad. A general upper bound for the satisfiability threshold of random $r$-sat formulae. Journal of Algorithms, 24:395-420, 1997.

[8] P. E. Dunne and M. Zito. An improved upper bound on the non-3-colourability threshold. Information Processing Letters, 65:17-23, 1998.

[9] P. Erdös and A. Rényi. On the evolution of random graphs. Publication of the Mathematical Institute of the Hungarian Academy of Sciences, 5:17-61, 1960.

[10] N. Fountoulakis. D.Phil. Thesis. In preparation.

[11] N. Fountoulakis. On the upper bound of the non-3-colourability threshold of random graphs. MSc Dissertation, Mathematical Institute, University of Oxford, 2000.

[12] I. Giotis, A. C. Kaporis, and L. M. Kirousis. Corrigendum to "A note on the non-colourability threshold of a random graph". Electronic Journal of Combinatorics, 7 (1), R29, 2000.

[13] T. Hogg and C. P. Williams. The hardest constrained problems: a double phase transition. Artificial Inteligence, 69:359-377, 1994.

[14] A. C. Kaporis, L. M. Kirousis, and Y. C. Stamatiou. A note on the non-colourability threshold of a random graph. Electronic Journal of Combinatorics, 7 (1), R29, 2000.

[15] L. M. Kirousis, E. Kranakis, D. Krizanc, and Y. C. Stamatiou. Approximating the unsatisfiability threshold of random formulas. Random Structures \& Algorithms, 12(3):253-269, 1998.

[16] C.J.H. McDiarmid. On the method of bounded differences. In J. Siemons, editor, Surveys in Combinatorics, pages 148-188. Cambridge University Press, 1989.

[17] M. Molloy. The chromatic number of sparse random graphs. M. Math. Thesis, University of Waterloo, 1992.

[18] M. Molloy. Thresholds for colourability and satisfiability in random graphs and boolean formulae. In J. Hirschfeld, editor, Surveys in Combinatorics, pages 166-200. Cambridge University Press, 2001.

[19] G. Strang. Linear Algebra and its Applications. Harcourt Brace Jovanovich, San Diego, 1988.

[20] N. M. Temme. Asymptotic estimates for Stirling numbers. Stud. Appl. Math., 89:223-243, 1993.

[21] R. Webster. Convexity. Oxford University Press, New York, 1994. 


\section{Appendices}

\section{A A note on Subsection 3.3}

We set $u=r / t$. Recall that $x_{0}(u)=x_{0}(r, t)$ is the root of the following equation:

$$
E_{u}(x)=1-e^{-x}-u x=0,
$$

for $0<u=u(r, t) \leq 1$. Let $x_{0}=x_{0}(u)$ throughout this Appendix. Suppose that $y$ is either $r$ or $t$. We have

$$
e^{-x_{0}} \frac{\partial x_{0}}{\partial y}-\frac{\partial u}{\partial y} x_{0}-u \frac{\partial x_{0}}{\partial y}=0
$$

Therefore,

$$
\frac{\partial x_{0}}{\partial y}=\frac{(\partial u / \partial y) x_{0}}{e^{-x_{0}}-u}
$$

Again, using (24) we have

$$
e^{x_{0}}-1-e^{x_{0}} u x_{0}=0 .
$$

So,

$$
u=e^{-x_{0}}\left(e^{x_{0}}-1\right) x_{0}^{-1}
$$

Thus, (25) becomes

$$
\begin{aligned}
\frac{\partial x_{0}}{\partial y} & =\frac{(\partial u / \partial y) x_{0}}{e^{-x_{0}}-e^{-x_{0}}\left(e^{x_{0}}-1\right) x_{0}^{-1}} \\
& =\frac{e^{x_{0}}(\partial u / \partial y) x_{0}^{2}}{1+x_{0}-e^{x_{0}}} .
\end{aligned}
$$

The denominator is negative, since $1+x_{0}<e^{x_{0}}$. Thus, the sign of this expression depends upon the sign of $\partial u / \partial y$. We shall use these expressions for the derivatives of $x_{0}(u)$ with respect to the variables on which it depends in Appendix Q.

Now, we shall give a lower bound on $f(t, r)$ and we will study its monotonicity. In fact we shall work with

$$
f(t, r)^{2}=f(u)^{2}=\frac{1-u}{x_{0}-\frac{1}{u}+1}
$$

where $u=r / t$. We have

$$
f(u)^{2}=\frac{1-u}{x_{0}-\frac{1}{u}+1} \frac{u(1-u)}{u x_{0}-(1-u)}>\frac{u(1-u)}{u x_{0}}=\frac{1-u}{x_{0}} .
$$

Thus,

$$
f(u)^{2}>\frac{1-u}{x_{0}} .
$$

In what follows, we are trying to investigate the monotonicity of the latter function. The derivative of this with respect to $u$ is

$$
\left(\frac{1-u}{x_{0}}\right)^{\prime}=\frac{-x_{0}-(1-u) \frac{\partial x_{0}}{\partial u}}{x_{0}^{2}} .
$$

We have to determine the sign of the numerator. Using (25), we obtain

$$
\begin{aligned}
-x_{0}-(1-u) \frac{\partial x_{0}}{\partial u} & =-x_{0}\left(1+(1-u) \frac{1}{e^{-x_{0}}-u}\right)=-x_{0}\left(1+(1-u) \frac{1}{1-u x_{0}-u}\right) \\
& =-x_{0}\left(1+\frac{1}{1-\frac{u}{1-u} x_{0}}\right) .
\end{aligned}
$$


Since $x_{0} \neq 0$, the expression on the right hand side is 0 if and only if $x_{0}=2(1-u) / u$. That is

$$
1-e^{-\frac{2(1-u)}{u}}-2(1-u)=0
$$

But the function $1-e^{-\frac{2(1-u)}{u}}-2(1-u)$ is strictly less than zero for $u<1$, and its only root in $(0,1]$ is $u=1$. This follows from the monotonicity of $1-e^{-\frac{2(1-u)}{u}}-2(1-u)$ with respect to $u$. Note, also, that for $u=\left(1-e^{-2}\right) / 2$, we have $x_{0}=2$. Since $u<1 / 2$, the monotonicity of $x_{0}$ with respect to $u$ implies that $x_{0}(1 / 2)<2$. This observation along with the above result yield $x_{0}>2(1-u) / u$, which implies that $1+\frac{1}{1-\frac{u}{1-u} x_{0}}<0$, for $0<u<1$. Hence, the derivative of $(1-u) / x_{0}$ with respect to $u$ is positive, and, therefore, this is a strictly increasing function with respect to $u$. Thus, for $u \leq 1$, this is an increasing function. We will use this fact to obtain a lower bound for $f(t, r)$ for specific $t, r$.

\section{B Some calculations from Subsection 3.4}

In this Appendix we present the elementary but tedious calculations for the function $P^{\prime}(k, \alpha, \lambda)$ on $\mathcal{D}$, as it was defined in Subsection B.4:

$$
\begin{aligned}
P^{\prime}(k, \alpha, \lambda)= & \\
= & \frac{n^{(2-\alpha-k)}(1-\alpha)^{(1-\alpha)}(1-k)^{(1-k)} e^{-(2-\alpha-k)}}{n^{\frac{\theta}{2}}(1-k)^{\frac{\theta}{2}}} 2^{\frac{\theta}{2} H(\lambda)}\left(\frac{1-k}{1-\alpha}\right)^{\frac{\lambda \theta}{2}} \times \\
& n^{\left(\frac{\theta \lambda}{2}+\alpha-1\right)} t(1-\alpha, \lambda \theta / 2) n^{\left(\frac{\theta}{2}-\frac{\theta \lambda}{2}-1+k\right)} t(1-k,(1-\lambda) \theta / 2) \times \\
& f\left(\frac{2(1-\alpha)}{\theta \lambda}\right) f\left(\frac{2(1-k)}{\theta(1-\lambda)}\right)\left(\frac{(1-k)(k-\alpha)}{\phi(k, \alpha)}\right)^{\frac{\theta}{2}(1-\lambda)}\left(1-\frac{(1-k)(k-\alpha)}{\phi(k, \alpha)}\right)^{\frac{\lambda \theta}{2}} \\
= & \frac{(1-\alpha)^{(1-\alpha)}(1-k)^{(1-k)} e^{-(2-\alpha-k)} 2^{\frac{\theta}{2} H(\lambda)}\left(\frac{1-k}{1-\alpha}\right)^{\frac{\lambda \theta}{2}} f\left(\frac{2(1-\alpha)}{\theta \lambda}\right) f\left(\frac{2(1-k)}{\theta(1-\lambda)}\right) \times}{(1-k)^{\frac{\theta}{2}}} \\
& t(1-\alpha, \lambda \theta / 2) t(1-k,(1-\lambda) \theta / 2)\left(\frac{(1-k)(k-\alpha)}{\phi(k, \alpha)}\right)^{\frac{\theta}{2}(1-\lambda)}\left(1-\frac{(1-k)(k-\alpha)}{\phi(k, \alpha)}\right)^{\frac{\lambda \theta}{2}},
\end{aligned}
$$

where

$$
t(x, y)=\left(e^{x_{0}}-1\right)^{y}\left(x_{0}\right)^{-x} e^{-(x-y)}(x-y)^{(x-y)}\left(\frac{x}{y}\right)^{y}\left(\frac{x}{x-y}\right)^{(x-y)}
$$

where $x_{0}=x_{0}(x / y)$. Thus, for $(k, \alpha, \lambda) \in \mathcal{D}$ we have

$$
\begin{aligned}
P^{\prime}(k, \alpha, \lambda)= & \\
= & \frac{(1-\alpha)^{(1-\alpha)}(1-k)^{(1-k)} e^{-(2-\alpha-k)}}{(1-k)^{\frac{\theta}{2}}} 2^{\frac{\theta}{2} H(\lambda)}\left(\frac{1-k}{1-\alpha}\right)^{\frac{\lambda \theta}{2}}\left(e^{x_{1}}-1\right)^{(1-\alpha)}\left(x_{1}\right)^{-\frac{\lambda \theta}{2}} e^{-\left(\frac{\theta \lambda}{2}+\alpha-1\right)} \times \\
& \left(\frac{\theta \lambda}{2}+\alpha-1\right)^{\left(\frac{\theta \lambda}{2}+\alpha-1\right)}\left(\frac{\lambda \theta}{2(1-\alpha)}\right)^{(1-\alpha)}\left(\frac{\lambda \frac{\theta}{2}}{\frac{\theta \lambda}{2}+\alpha-1}\right)^{\left(\frac{\theta \lambda}{2}+\alpha-1\right)} \times \\
& \left(e^{x_{2}}-1\right)^{(1-k)}\left(x_{2}\right)^{-\frac{\theta}{2}(1-\lambda)} e^{-\left(\frac{\theta}{2}-\frac{\theta \lambda}{2}-1+k\right) \times} \times\left(\frac{\theta(1-\lambda)}{2}\right)^{\left(\frac{\theta}{2}-\frac{\theta \lambda}{2}-1+k\right)} \times \\
& \left.\left(\frac{\theta}{2}-\frac{\theta \lambda}{2}-1+k\right)^{\left(\frac{\theta}{2}-\frac{\theta \lambda}{2}-1+k\right)}\left(\frac{\theta(1-\lambda)}{2(1-k)}\right)^{(1-k)}(1+k)\right)^{\frac{\theta}{2}(1-\lambda)}\left(1-\frac{(1-k)(k-\alpha)}{\phi(k, \alpha)}\right)^{\frac{\lambda \theta}{2}} \\
& f\left(\frac{2(1-\alpha)}{\theta \lambda}\right) f\left(\frac{2(1-k)}{\theta(1-\lambda)}\right)\left(\frac{(1-k)(k-\alpha)}{\phi(k, \alpha)}\right)^{\frac{(1-\lambda)}{(1-\alpha)}}
\end{aligned}
$$




$$
\begin{aligned}
= & (1-k)^{-\frac{\theta}{2}} 2^{\frac{\theta}{2} H(\lambda)}\left(\frac{1-k}{1-\alpha}\right)^{\frac{\lambda \theta}{2}}\left(e^{x_{1}}-1\right)^{n(1-\alpha)}\left(e^{x_{2}}-1\right)^{(1-k)}\left(x_{1}\right)^{-\frac{\lambda \theta}{2}}\left(x_{2}\right)^{-\frac{\theta}{2}(1-\lambda)} e^{-\frac{\theta}{2}} \times \\
& \left(\frac{\lambda \theta}{2}\right)^{\frac{\theta \lambda}{2}}\left(\frac{\theta(1-\lambda)}{2}\right)^{\frac{\theta(1-\lambda)}{2}} f\left(\frac{2(1-\alpha)}{\theta \lambda}\right) f\left(\frac{2(1-k)}{\theta(1-\lambda)}\right) \times \\
& \left(\frac{(1-k)(k-\alpha)}{\phi(k, \alpha)}\right)^{\frac{\theta}{2}(1-\lambda)}\left(1-\frac{(1-k)(k-\alpha)}{\phi(k, \alpha)}\right)^{\frac{\lambda \theta}{2}},
\end{aligned}
$$

where $x_{1}=x_{0}\left(\frac{2(1-\alpha)}{\theta \lambda}\right)$, i.e. it is the positive root of the equation

$$
1-e^{-x}-\frac{2(1-\alpha)}{\theta \lambda} x=0
$$

and $x_{2}=x_{0}\left(\frac{2(1-k)}{\theta(1-\lambda)}\right)$, i.e. it is the positive root of the equation

$$
1-e^{-x}-\frac{2(1-k)}{\theta(1-\lambda)} x=0
$$

\section{The concavity of $h(k, \alpha, \lambda)$ over $\mathcal{D}$}

In this Appendix, we show that the function $h(k, \alpha, \lambda)$ as it was defined in Section 5 is strictly concave over the interior of $\mathcal{D}$, where $\mathcal{D}$ was defined in (17). (Recall that the function $h(k, \alpha, \lambda, \theta)$ was defined in (18), and we have fixed $\theta=\theta_{2}=4.9895$ to obtain $h(k, \alpha, \lambda)$.) This treatment improves the proof in an earlier version of this paper; and was inspired by the correction [12] to [14]. We split the function $h(k, \alpha, \lambda)$ (multiplied by $\ln 2$ to change to natural logarithms) into four parts. Namely, for any $(k, \alpha, \lambda) \in \mathcal{D}$, we have

$$
\ln 2 h(k, \alpha, \lambda)=h_{1}(k, \alpha, \lambda)+h_{2}(k, \alpha, \lambda)+h_{3}(k, \alpha, \lambda)+\frac{\theta_{2}}{2} h_{4}(k, \alpha, \lambda),
$$

where

$$
\begin{aligned}
& h_{1}(k, \alpha, \lambda)=-(1-k) \ln (1-k)-(k-\alpha) \ln (k-\alpha)-\alpha \ln \alpha+\left(\frac{\theta_{2}}{2}-\frac{\theta_{2}}{2} \log (e)+\frac{\theta_{2}}{2} \log \left(\frac{\theta_{2}}{2}\right)\right) \ln 2, \\
& h_{2}(k, \alpha, \lambda)=(1-k) \ln \left(e^{x_{2}}-1\right)-\frac{\theta_{2}}{2}(1-\lambda) \ln x_{2}-\frac{\theta_{2}(1-\lambda)}{2} \ln (1-k)+\frac{\theta_{2}(1-\lambda)}{2} \ln (1-\lambda), \\
& h_{3}(k, \alpha, \lambda)=(1-\alpha) \ln \left(e^{x_{1}}-1\right)-\frac{\lambda \theta_{2}}{2} \ln x_{1}-\frac{\lambda \theta_{2}}{2} \ln (1-\alpha)+\frac{\lambda \theta_{2}}{2} \ln \lambda, \\
& h_{4}(k, \alpha, \lambda)=-\lambda \ln \lambda-(1-\lambda) \ln (1-\lambda)+(1-\lambda) \ln (1-k)+(1-\lambda) \ln (k-\alpha)+\lambda \ln \alpha+\lambda \ln (1-\alpha),
\end{aligned}
$$

and $x_{1}, x_{2}$ are defined in (28) and (29), respectively. For $i=1, \ldots, 4$, we set $h_{i}=h_{i}(k, \alpha, \lambda)$. We prove that each of these functions is concave over the interior of $\mathcal{D}$, with $h_{4}$ being strictly concave there. To prove that a suitably differentiable function is concave (strictly concave, respectively) over an open domain, we have to prove that its Hessian matrix (i.e. the matrix of the second partial derivatives) is negative semidefinite (definite, respectively) over this domain (see for example [2]], Theorem 5.5.5 p. 230). By, for example, Theorem 6E in [19] (p.339), to check negative semidefiniteness (definiteness, respectively) of a real symmetric matrix it is necessary and sufficient to show that the principal minors have alternating signs (and are non-zero in the case of definiteness), the first one being non-positive. Thus, we may deal with $h_{1}, \ldots, h_{4}$ as follows (for further details of the calculations see [10]).

1. Observe that $h_{1}$ does not depend on $\lambda$. It can be easily checked that it is concave over $\mathcal{D}$. The elements of the Hessian matrix with respect to $k$ and $\alpha$ are the following:

$$
\frac{\partial^{2} h_{1}}{\partial k^{2}}=-\frac{1}{(1-k)}-\frac{1}{(k-\alpha)}<-\frac{1}{(k-\alpha)},
$$




$$
\begin{aligned}
\frac{\partial^{2} h_{1}}{\partial k \partial \alpha} & =\frac{1}{k-\alpha} \\
\frac{\partial^{2} h_{1}}{\partial \alpha^{2}} & =-\frac{1}{\alpha}-\frac{1}{k-\alpha}<-\frac{1}{(k-\alpha)} .
\end{aligned}
$$

Thus $\frac{\partial^{2} h_{1}}{\partial k^{2}}<0$ and $\frac{\partial^{2} h_{1}}{\partial k^{2}} \frac{\partial^{2} h_{1}}{\partial \alpha^{2}}-\left(\frac{\partial^{2} h_{1}}{\partial k \partial \alpha}\right)^{2}>0$, and so is concave over $\mathcal{D}$.

2. Next we consider the function $h_{2}$, which does not depend on $\alpha$. After doing some algebraic manipulations and using (29) and (26), we obtain:

$$
\frac{\partial^{2} h_{2}}{\partial k^{2}}=\frac{\theta_{2}}{2} \frac{1-\lambda}{(1-k)^{2}} \frac{x_{2}}{1+x_{2}-e^{x_{2}}} .
$$

Similarly,

$$
\frac{\partial^{2} h_{2}}{\partial \lambda^{2}}=\frac{\theta_{2}}{2(1-\lambda)} \frac{x_{2}}{1+x_{2}-e^{x_{2}}}
$$

and

$$
\frac{\partial^{2} h_{2}}{\partial k \partial \lambda}=-\frac{\theta_{2}}{2} \frac{1}{1-k} \frac{x_{2}}{1+x_{2}-e^{x_{2}}} .
$$

Clearly we have $\frac{\partial^{2} h_{2}}{\partial k^{2}}<0$ and it is easily checked from the above that $\frac{\partial^{2} h_{2}}{\partial k^{2}} \frac{\partial^{2} h_{2}}{\partial \lambda^{2}}-\left(\frac{\partial^{2} h_{2}}{\partial k \partial \lambda}\right)^{2}$ is identically 0 over $\mathcal{D}$; and so $h_{2}$ is concave there.

3. The function $h_{3}$ has precisely the same form as $h_{2}$, where $1-\lambda$ has been replaced by $\lambda$. Thus, we deduce that $h_{3}$ is also concave over the interior of $\mathcal{D}$.

4. We show that $h_{4}$ is strictly concave over the interior of $\mathcal{D}$. Its Hessian matrix is:

$$
\left[\begin{array}{lll}
\frac{\partial^{2} h_{4}}{\partial k^{2}} & \frac{\partial^{2} h_{4}}{\partial k \partial \alpha} & \frac{\partial^{2} h_{4}}{\partial k \partial \lambda} \\
\frac{\partial^{2} h_{4}}{\partial k \partial \alpha} & \frac{\partial^{2} h_{4}}{\partial \alpha^{2}} & \frac{\partial^{2} h_{4}}{\partial \alpha \partial \lambda} \\
\frac{\partial^{2} h_{4}}{\partial k \partial \lambda} & \frac{\partial^{2} h_{4}}{\partial \alpha \partial \lambda} & \frac{\partial^{2} h_{4}}{\partial \lambda^{2}}
\end{array}\right]=\left[\begin{array}{ccc}
-\frac{1-\lambda}{(1-k)^{2}}-\frac{1-\lambda}{(k-\alpha)^{2}} & \frac{1-\lambda}{(k-\alpha)^{2}} & \frac{1}{1-k}-\frac{1}{k-\alpha} \\
\frac{1-\lambda}{(k-\alpha)^{2}} & -\frac{1-\lambda}{(k-\alpha)^{2}}-\frac{\lambda}{\alpha^{2}}-\frac{\lambda}{(1-\alpha)^{2}} & \frac{1}{k-\alpha}+\frac{1}{\alpha}-\frac{1}{1-\alpha} \\
\frac{1}{1-k}-\frac{1}{k-\alpha} & \frac{1}{k-\alpha}+\frac{1}{\alpha}-\frac{1}{1-\alpha} & -\frac{1}{\lambda}-\frac{1}{1-\lambda}
\end{array}\right] .
$$

To show strict concavity, it suffices to show that the principal minors are non-zero and have alternating signs with the first one being negative. It is easy to see that the first two principal minors are as required, since $\frac{\partial^{2} h_{4}}{\partial k^{2}}$ and $\frac{\partial^{2} h_{4}}{\partial \alpha^{2}}$ are strictly less than $-\frac{1-\lambda}{(k-\alpha)^{2}}$. The third principal minor, which is the determinant $\Delta$ of the matrix, satisfies

$$
\Delta=\frac{-(1-\lambda) \alpha^{2}(1-\alpha)^{2}+4 \lambda \alpha(1-\alpha)(k-\alpha)(1-k)-2 \lambda^{2}(k-\alpha)(1-k)}{\lambda \alpha^{2}(1-\alpha)^{2}(k-\alpha)^{2}(1-k)^{2}},
$$

and this can verified e.g. by using Maple (or see [10] for the details). The denominator is always strictly positive, so it is sufficient to show that the numerator in negative for any $(k, \alpha, \lambda) \in \mathcal{D}$. Let us fix $\alpha, \lambda<1$ and express the numerator as a quadratic function of $k$, for $\alpha \leq k \leq 1$. We obtain $-k^{2} \eta+k(\alpha+1) \eta-\left((1-\lambda) \alpha^{2}(1-\alpha)^{2}+\alpha \eta\right)$, where $\eta=(4 \alpha(1-\alpha)-2 \lambda) \lambda$. If $\eta=0$, then obviously this expression is negative. On the other hand, for $k=\alpha$ or $k=1$, the above expression is equal to $-(1-\lambda) \alpha^{2}(1-\alpha)^{2}<0$. So, to show that this quadratic function is negative it is sufficient to prove that at its stationary point $k=(\alpha+1) / 2$ this is negative. But the value of the function at this point is

$$
-(1-\lambda) \alpha^{2}(1-\alpha)^{2}+\lambda \alpha(1-\alpha)^{3}-\frac{\lambda^{2}(1-\alpha)^{2}}{2}=-(1-\alpha)^{2}\left(\frac{\alpha^{2}}{2}+\frac{1}{2}(\alpha-\lambda)^{2}\right)<0,
$$

and this concludes the proof of the strict concavity of $h_{4}$ over $\mathcal{D}$. 
